\title{
Optical coherence tomography angiography for the measurement of optic disc: Macular relationship
}

European Journal of Ophthalmology I-5

(C) The Author(s) 2020 Article reuse guidelines: sagepub.com/journals-permissions DOI: I0.1 I77/II20672I 20904633 journals.sagepub.com/home/ejo

(S)AGE

\author{
Massimiliano Serafino ${ }^{1,2}$, Matteo Scaramuzzi ${ }^{1,2}$ (D), \\ Francesco Bonsignore $^{1,2}$, Lucia Vitale ${ }^{1,2}$, Adriano Magli ${ }^{3}$ \\ and Paolo Nucci ${ }^{1,2}$
}

\begin{abstract}
Introduction: Fundus photography is the gold standard for assessing ocular torsion over the last 30 years. However, it is not a precise and reproducible tool during clinical practice. Optical coherence tomography angiography is characterized by precise identification of the macula and the optic disc, and it could be an effective method to easily calculate the angle of ocular torsion, compared to fundus photography. The aim of this study was to show whether any difference in the measurement and the accuracy of the angle of torsion between the head of the optic nerve and the fovea was present.

Methods: This is a prospective single-, referral-center study conducted at the San Giuseppe Hospital in Milan on 80 eyes of 40 adult patients, included in a random-sample way. Exclusion criteria were non-cooperation, higher refractive errors of \pm 3 diopters, retinal and optic disc pathologies, and ocular movement disorders.

Results: Patients' mean age was $54.3 \pm 16.3$ (range: $22-83$ ) years. The angle measured by the fundus camera was $7.78^{\circ} \pm 3.04^{\circ}$, while the angle measured by the angiography was $7.09^{\circ} \pm 3.08^{\circ}(p=.035)$. The mean interocular difference was $1.54^{\circ} \pm 3.42^{\circ}$ for fundus photography and $0.5^{\circ} \pm 4.71^{\circ}$ for angiography $(p=.013)$.

Conclusion: Optical coherence tomography angiography is a very useful, fast, precise, reproducible, and reliable technique in cooperative subjects, not inferior to the fundus camera and less prone to human error.
\end{abstract}

\section{Keywords}

Optics/refraction/instruments, diagnostic techniques, strabismus, eye movement disorders, vertical and A/V patterns

Date received: 23 August 2019; accepted: 2 January 2020

\section{Introduction}

Different ways of defining the precise relative position of the optic nerve head and macula have been studied in the past. It would be important to have access to a method to easily calculate their relative location in order to determine the angle between the fovea and the optic disc. This is particularly useful for obtaining a value of the ocular torsion.

There are several subjective methods of evaluating the extent of ocular torsion, also known as cyclo-position. Examples include Double Maddox's rod test, Bagolini's eyeglasses, indirect ophthalmoscopic lens, slit lamp biomicroscopy, perimetry, or synoptophore. However, obtaining reliable, standardized, and reproducible results using these subjective measurement methods remains complicated.
In 1982, the first group that objectively studied the anatomical angular relationship between the optic disc and the macula used fundus photography. ${ }^{1}$ They analyzed a group of 50 non-strabismic volunteers and found that the normal foveal position corresponded to one-third of the disc

'Department of Clinical Sciences and Community Health, University of Milan, Milan, Italy

${ }^{2}$ University Eye Clinic, San Giuseppe Hospital, IRCCS MultiMedica, Milan, Italy

${ }^{3}$ Department of Ophthalmology, Orthoptics and Pediatric

Ophthalmology, University of Salerno, Salerno, Italy

Corresponding author:

Matteo Scaramuzzi, University Eye Clinic, San Giuseppe Hospital, IRCCS MultiMedica, Via San Vittore I2, 20123 Milan, Italy.

Email: matteo_scaramuzzi@hotmail.com 
diameter below a horizontal line passing through the center of the optic nerve head. The following year, the same group showed the usefulness of fundus photography for documenting ocular torsion in patients with vertical strabismus. $^{2}$

More recently, other authors have measured foveal disc angle in different patient groups, but they differed significantly. ${ }^{3,4}$ Therefore, even though it was considered the gold standard for ocular torsion measurement, ${ }^{5}$ fundus photography should not be considered a reproducible tool without a standardized approach.

The optic disc proved to be easier to be located than the fovea. This is due to its brightness, location, and the presence of prominent blood vessels, although accurate segmentation can be difficult. ${ }^{6,7}$

Conversely, the detection of fovea could be much more difficult because it has no specific characteristics. In order to detect it easily, several authors have proposed their techniques, such as semi-automated analysis, statistical analysis of metrics and pixel intensity, mathematical morphology, or blur scale variations, among others. ${ }^{8-11}$

Since these methods are difficult to apply in clinical practice, other solutions must be found. In a recent case report of two patients, Sophocleous demonstrated that fundus torsion could be demonstrated with the use of optical coherence tomography (OCT). ${ }^{12}$ In relation to the other methods proposed, this method takes less time, no dilation of the pupil is necessary, and it is less prone to human errors, because the macula transverse section reveals precisely the location of the fovea. ${ }^{12}$

OCT angiography (OCTA) is a new technology that further enhances the role of imaging. It allows analyzing the retinal and choroidal vascular system without injecting the dye. OCTA instruments take advantage of the innovations included in the original OCT, such as eye tracking systems, fovea computer detection, or rapid acquisition speed, by adding improved anatomical structure capabilities, such as for vascularization of the head optic nerve or foveal avascular zone. ${ }^{13-15}$

In our study, we compared the gold standard method for measuring ocular torsion, fundus photography, with the latest innovation in the field of retinal imaging, OCTA, in a sample of healthy subjects.

\section{Materials and methods}

We included in our study 40 patients ( 80 eyes). The patients were examined at the San Giuseppe Hospital in Milan. This study was conducted in accordance with the Helsinki Declaration, and the study protocol was approved by our governing board.

Patients were informed of the procedure and agreed to obtain fundus photographs and OCTA images. All patients underwent a complete ophthalmological examination. The exclusion criteria were as follows: inappropriate cooperation, a corneal or crystalline disorder that made examinations difficult, abnormal ocular movement, ocular deviation, retinal pathologies, pathologies of the optic nerve, amblyopia, myopia greater than -3 diopters, hyperopia greater than +3 diopters, and astigmatism greater than \pm 1 diopter. All patients with none of these characteristics were included. Only one of the authors (F.B.) was responsible for all the measurements made, in order to exclude any bias between operators. We paid particular attention to the alignment of the patient's head on the lateral marks and the correct placement of the chin. We use a precision level with a balancing air bubble positioned on the patent's head to be sure that the patient maintained the correct position.

The fundus photography was done with a CR-2 fused camera (Canon, Japan). We obtained $50^{\circ}$ photographs to visualize the macula and the optic nerve on the same frame. The angle was measured between a horizontal line passing through the fovea and a line connecting the fovea to the geometric center of the optic nerve head (Figure 1).

We used Cirrus OCTA (Carl Zeiss Meditec, Jena, Germany) to obtain OCTA images. We obtained an image of $6 \mathrm{~mm} \times 6 \mathrm{~mm}$ in order to visualize the macula and the optic nerve on the same frame. The precise location of the fovea was obtained through the OCT analysis manually moving the horizontal and vertical lines until they passed through the fovea. The angle was measured between the horizontal line passing through the fovea and the line connecting the fovea to the center of the major vascularization of the optic nerve (Figure 2).

The angle was calculated using ImageJ software version 1.40 (National Institutes of Health, Bethesda, MD, USA).

SPSS Statistics 17.0 software was used for statistical analysis (SPSS, Inc., Chicago, IL, USA). We selected a group sample sizes of 80 eyes and 40 patients in order to achieve an $80 \%$ power to reject the null hypothesis of equal means with a significance level of $5 \%$. Values of $\mathrm{p}<.05$ were considered significant. The nonparametric Wilcoxon test was used to compare measured angles with OCTA and a fundus camera.

\section{Results}

The patients' mean age was $54.3 \pm 16.3$ (range: $22-83$ ) years; 23 of them were female $(57.5 \%)$.

Taking into account all eyes, the angle measured by the fundus camera was $7.78^{\circ} \pm 3.04^{\circ}$, while the angle measured by the OCTA was $7.09^{\circ} \pm 3.08^{\circ}(\mathrm{p}=.035)$. If the analysis is limited to the right eye, the measured angle is, respectively, $8.55^{\circ} \pm 2.95^{\circ}$ and $7.34^{\circ} \pm 2.84^{\circ}(\mathrm{p}=.005)$. In contrast, left eye analysis showed that the angles were not significantly different, $7.01^{\circ} \pm 2.98^{\circ}$ versus $6.84^{\circ} \pm 3.34^{\circ}$ $(\mathrm{p}=.8)($ see Table 1).

The mean interocular difference was $1.54^{\circ} \pm 3.42^{\circ}$ for fundus photography and $0.5^{\circ} \pm 4.71^{\circ}$ for OCTA $(p=.013)$. 


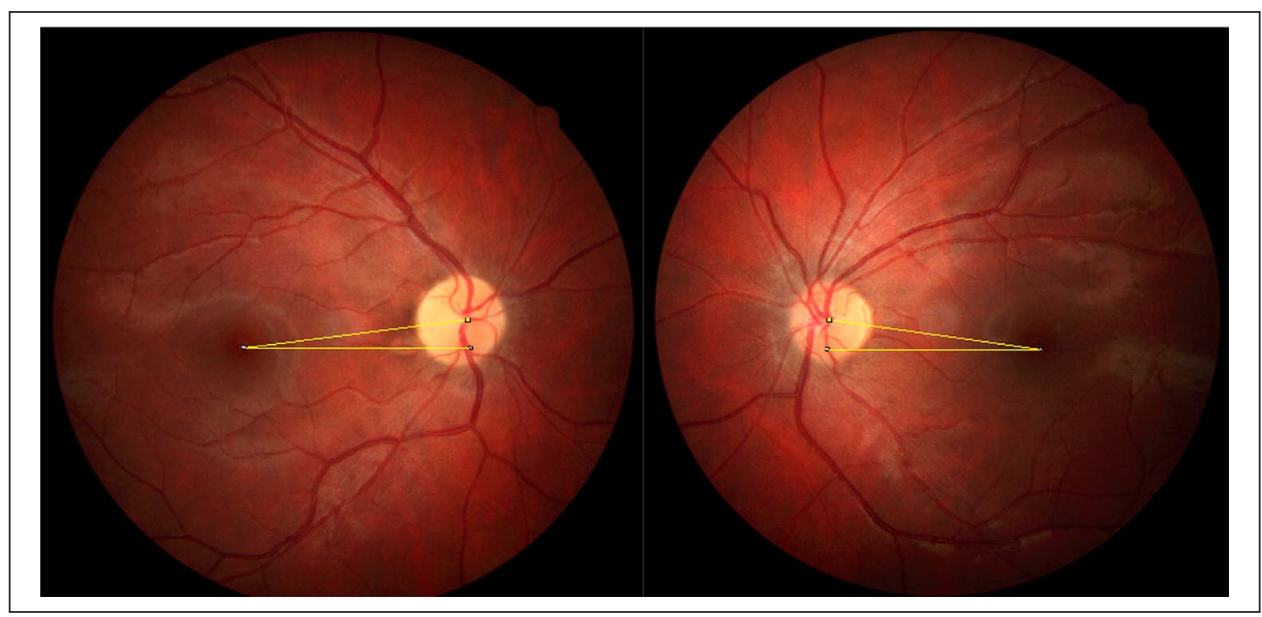

Figure I. Fundus photography of patient I. The angle was calculated between the horizontal line passing through the fovea and the inclined line that connects the fovea to the geometric center of the optic nerve head.

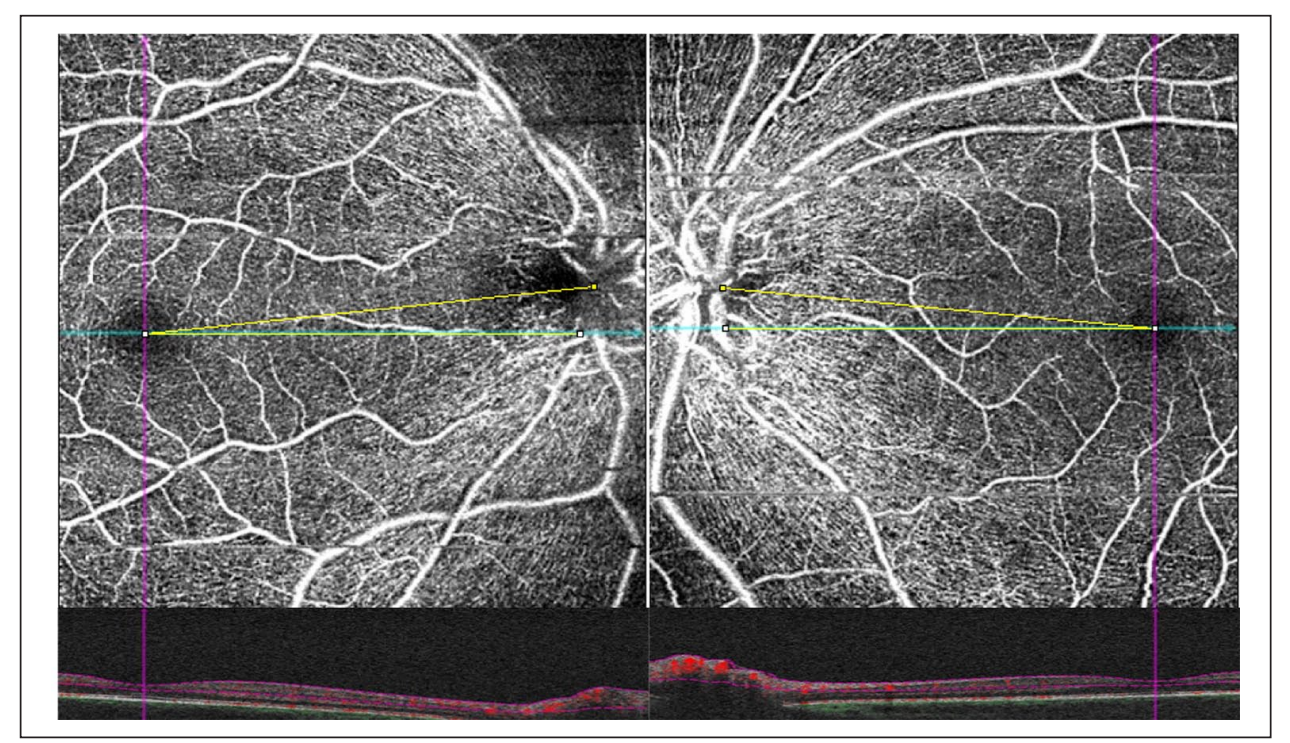

Figure 2. OCTA of patient I. The angle was calculated between the horizontal line passing through the localized fovea through the OCT scan and the inclined line that connects the fovea to the center of the major vascularization of the optic nerve.

Table I. Values of optic nerve head — fovea angle measured with fundus photography and OCT angiography.

\begin{tabular}{llll}
\hline & The angle measured with fundus photography & The angle measured with OCT angiography & P value \\
\hline All eyes & $7.78^{\circ} \pm 3.04^{\circ}$ & $7.09^{\circ} \pm 3.08^{\circ}$ & .035 \\
Right eyes & $8.55^{\circ} \pm 2.95^{\circ}$ & $7.34^{\circ} \pm 2.84^{\circ}$ & .005 \\
Left eyes & $7.01^{\circ} \pm 2.98^{\circ}$ & $6.84^{\circ} \pm 3.34^{\circ}$ & .8 \\
\hline
\end{tabular}

OCT: optical coherence tomography.

\section{Discussion}

In our study, the foveal optic head angle was $7.78^{\circ} \pm 3.04^{\circ}$ and $7.09^{\circ} \pm 3.08^{\circ}$ using, respectively, fundus photography and OCTA. Bixenman and von Noorden ${ }^{1}$ found an angle of $7.2^{\circ} \pm 2.5^{\circ}$ in 100 eyes of 50 patients in their first study in which they used fundus photography in 1982. Lefevre et al. ${ }^{4}$ analyzed 150 patients divided into three different age groups, without angular variations, with an average value of $6.3^{\circ} \pm 3.4^{\circ}$. The largest sample of patients was collected by Williams and Wilkinson, ${ }^{16} 446$ eyes with a mean angle of $6.1^{\circ} \pm 3.3^{\circ}$. Kothari et al. ${ }^{17}$ described a lower value in a 
Table 2. Different values of optic nerve head—fovea angle measured with fundus photography by previous authors.

\begin{tabular}{|c|c|c|}
\hline Authors & Number of eyes & The angle measured with fundus photography \\
\hline Bixenman and von Noorden' & 100 & $7.2^{\circ} \pm 2.5^{\circ}$ \\
\hline Williams and Wilkinson ${ }^{16}$ & 446 & $6.1^{\circ} \pm 3.3^{\circ}$ \\
\hline Kothari et al. ${ }^{17}$ & 72 & $6.1^{\circ} \pm 4.3^{\circ}$ \\
\hline Lefevre et al. ${ }^{4}$ & 300 & $6.3^{\circ} \pm 3.4^{\circ}$ \\
\hline Freedman et al. ${ }^{18}$ & 10 & $4.8^{\circ}$ \\
\hline
\end{tabular}

subset of 72 eyes (36 patients): $5.5^{\circ} \pm 3.3^{\circ}$ using a slit lamp stereoscopic technique and $6.1^{\circ} \pm 4.3^{\circ}$ with a photograph of fundus. Other authors have analyzed the angle of the optic nerve and fovea using a fundus camera, with a smaller number of patients (Table 2). ${ }^{18}$

The different values described by our work and the previous ones are difficult to compare because the sample of patients could be very different. However, not focusing on numerical values, but on qualitative values, even our results confirm the general role that describes the fovea located in an area between one-third of a disc diameter under a horizontal line through the center of the optic nerve head and the center of the head of the optic nerve itself.

Considering the mean interocular difference, the results we found using the fundus camera are similar to those described by Bixenman and von Noorden ${ }^{1}\left(1.6^{\circ} \pm 1.2^{\circ}\right)$, but lower than those proposed by Kothari et al. ${ }^{17}$ $\left(3.3^{\circ} \pm 2.7^{\circ}\right.$ and $5.5^{\circ} \pm 4.6^{\circ}$, respectively, measured with a slit lamp and photograph), but they included patients with various refractive errors. Jethani et al. ${ }^{19}$ studied the ocular torsion of a group of Indian children and found results similar to ours $\left(1.15^{\circ} \pm 1.39^{\circ}\right)$.

Looking at our results, the value of the disc-foveal angle obtained with the OCTA was significantly lower than that of the fundus photography, considering all the eyes and the right ones. An average difference of about only one degree was found. This finding could probably not change the relevance of the measurements during the clinical practice but show that OCTA is, at least, as reliable as photography in the measurement of ocular torsion.

More importantly, the interocular difference was significantly reduced if measured with OCTA. The possible explanation to the significative difference in measurement between the two instruments and the reduced interocular difference measured with the OCTA is related, in our opinion, to the OCT technology: in OCTA it is possible to precisely identify the center of the fovea, differently from fundus photography. So, this high level of precision allows the use of this technology to be extended in the field of ocular torsion, other than areas of retinal pathologies or optic nerve. In difficult and doubtful cases of incyclotorsion or excyclotorsion, the possibility of having OCTA could be useful for a precise measurement.

We found that there was no significant difference in the angle of the left eye as measured by both techniques. This result could be due to different average interocular values or the relatively small size of the sample. Another possibility could be the existence of a divergence in static torsion measurements under monocular or binocular observation conditions, but we could not measure the torsion while the contralateral eye maintains fixation for the size and the shape of the instruments, so a relative torsional alignment should be considered; however, this limitation impacts in the same way on both the techniques. Alternatively, the presence of a true variance in the population could really exist, as suggested by Guyton, ${ }^{20}$ who observed a wide range of physiological torsion in the normal population, up to $18^{\circ}$ between the two eyes.

Our study has some limitations, as it is a monocentric study. Further studies are also needed to test OCTA on a pathological sample of patients with head-tilted position or cyclotropia, possibly comparing it with double Maddox rod test, as well as fundus photography, before and after surgery. However, this is a homogeneous group of patients, and this is the first work that demonstrates the usefulness of the OCT angiographic technology in this area and its non-inferiority to the current gold standard method.

In conclusion, fundus photography should remain the gold standard technique for the measurement of the head of the optic nerve-foveal angle, and thus of the ocular torsion, thanks to a wider distribution. However, the OCTA is a very useful, fast, precise, reproducible, and reliable technique in cooperative subjects, not inferior to the fundus camera and less prone to human errors.

\section{Declaration of conflicting interests}

The author(s) declared the following potential conflicts of interest with respect to the research, authorship, and/or publication of this article: P.N. confirms he is an associate editor of this journal and was not involved in the peer review process for this paper.

\section{Funding}

The author(s) received no financial support for the research, authorship, and/or publication of this article.

\section{ORCID iDs}

Matteo Scaramuzzi iD https://orcid.org/0000-0003-2270-1732

Adriano Magli (iD https://orcid.org/0000-0002-6490-2068 


\section{References}

1. Bixenman WW and von Noorden GK. Apparent foveal displacement in normal subjects and in cyclotropia. Ophthalmology 1982; 89(1): 58-62.

2. Morton GV, Lucchese N and Kushner BJ. The role of funduscopy and fundus photography in strabismus diagnosis. Ophthalmology 1983; 90(10): 1186-1191.

3. Madigan WP Jr and Katz NN. Ocular torsion-direct measurement with indirect ophthalmoscope and protractor. $J$ Pediatr Ophthalmol Strabismus 1992; 29(3): 171-174.

4. Lefevre F, Leroy K, Delrieu B, et al. Study of the optic nerve head-fovea angle with retinophotography in healthy patients. J Fr Ophtalmol 2007; 30(6): 598-606.

5. Philips PH and Hunter DG. Evaluation of ocular torsion and principles of management. In: Santiago P and Rosenbaum $\mathrm{AL}$ (eds) Clinical strabismus management principles and surgical techniques. Philadelphia, PA: WB Saunders Company, 1999, pp. 52-72.

6. Niemeijer $M$, Abramoff $\mathrm{MD}$ and van Ginneken $B$. Segmentation of the optic disc, macula and vascular arch in fundus photographs. IEEE Trans Med Imaging 2007; 26(1): 116-127.

7. Pereira C, Goncalves L and Ferreira M. Optic disc detection in color fundus images using ant colony optimization. Med Biol Eng Comput 2013; 51(3): 295-303.

8. Ganesan K, Acharya RU, Chua CK, et al. Identification and localization of fovea on colour fundus images using blur scales. Proc IMechE, Part H: J Engineering in Medicine 2014; 228(9): 962-970.

9. Ahmad Fadzil MH, Izhar LI and Nugroho HA. Determination of foveal avascular zone in diabetic retinopathy digital fundus images. Comput Biol Med 2010; 40(7): 657-664.

10. Niemeijer M, Abramoff MD and van Ginneken B. Fast detection of the optic disc and fovea in color fundus photographs. Med Image Anal 2009; 13(6): 859-870.
11. Welfer D, Scharcanski J and Marinho DR. Fovea center detection based on the retina anatomy and mathematical morphology. Comput Meth Prog Biomed 2011; 104(3): 397-409.

12. Sophocleous S. Use of optical coherence topography for objective assessment of fundus torsion. BMJ Case Rep. Epub ahead of print 23 February 2017. DOI: 10.1136/bcr2016-216867.

13. Alnawaiseh M, Brand C, Bormann E, et al. Quantification of macular perfusion using optical coherence tomography angiography: repeatability and impact of an eye-tracking system. BMC Ophthalmol 2018; 18(1): 123.

14. Manalastas PIC, Zangwill LM, Saunders LJ, et al. Reproducibility of optical coherence tomography angiography macular and optic nerve head vascular density in glaucoma and healthy eyes. J Glaucoma 2017; 26(10): 851-859.

15. Carpineto P, Mastropasqua R, Marchini G, et al. Reproducibility and repeatability of foveal avascular zone measurements in healthy subjects by optical coherence tomography angiography. Br J Ophthalmol 2016; 100(5): 671-676.

16. Williams TD and Wilkinson JM. Position of the fovea centralis with respect to the optic nerve head. Optom Vis Sci 1992; 69(5): 369-377.

17. Kothari MT, Venkatesan G, Shah JP, et al. Can ocular torsion be measured using the slitlamp biomicroscope? Indian J Ophthalmol 2005; 53(1): 43-47.

18. Freedman SF, Gearinger MD, Enyedi LB, et al. Measurement of ocular torsion after macular translocation: disc fovea angle and Maddox rod. J AAPOS 2003; 7(2): 103-107.

19. Jethani J, Seethapathy G, Purohit J, et al. Measuring normal ocular torsion and its variation by fundus photography in children between 5-15 years of age. Indian J Ophthalmol 2010; 58(5): 417-419.

20. Guyton DL. Clinical assessment of ocular torsion. Am Orthopt J 1983; 33(1): 7-15. 